\title{
Using Flower Pollination Algorithm for Segmentation with Threesholding
}

\author{
Ibrahim M. El-Henawy \\ Faculty of computer and Information Sciences \\ Zagazig University \\ Zagazig, Egypt
}

\author{
Mohamed A. Fahmy \\ Computer Science Department Higher Technology \\ Institute \\ 10th of Ramadan City
}

\begin{abstract}
The bio-inspired algorithm was development to generate optimum threshold values for image segmentation the flower pollination algorithm (FPA) is one of heuristic and bioinspired algorithms that deals with continuous and combinatorial optimization problems and it can use for image segmentation of the image satellite. The satellite images are very hard to be segmented because the images are weakly correlated and the important regions are unclear. The purpose of the research is to use "FPA" in satellite image segmentation.
\end{abstract}

\section{General Terms}

Optimization algorithms, satellite image segmentation, flower pollination

\section{Keywords}

Flower pollination, segmentation, threesholding, Kapur's entropy

\section{INTRODUCTION}

The operation of getting "extracting "connected region or regions that have certain homogeneity "based on special feature or features in spectral component that are defined in specified color space model is called image segmentation.so it's important to say that the color space model is the key player in the process of image segmentation he plays the most important role [, from the definition of segmentation and the nature of the satellite image that have many ambiguous regions and small multiple regions of interest the pixel have weak local correlation it's difficult to decide the homogeneity measure witch can be used to recognize the objects and differentiate between them. The satellite images of agricultural lands are very difficult to segmented because of the poor of illumination, resolution and environmental conditions that prevail while capturing the images [2], bioinspired optimization algorithms are the algorithms that develop for solving the complex problems that have limited time and resources the algorithms is divided into three main types "evolution ,swarm based and ecology "every type of them have sub types this types can be demonstrated according to the area of inspiration from nature [3]. FPA was designed and developed by Xin-She Yang in 2013 the algorithm is based on the Flower pollination characteristics in the natural world. The pollination process, flower constancy and pollinator behavior as the following rules:

1.Biotic and cross-pollination is considered as global pollination process with pollen carrying pollinators performing Lévy flights.

2. Abiotic and self-pollination are considered as local pollination.
3. Flower constancy can be considered, as the reproduction probability is proportional to the similarity of two flowers involved

4. Local pollination and global pollination is controlled by a switch probability $\mathrm{p} \in[0,1]$. Due to the physical proximity and other factors such as wind, local pollination can have a significant fraction $p$ in the overall pollination activities. [4]

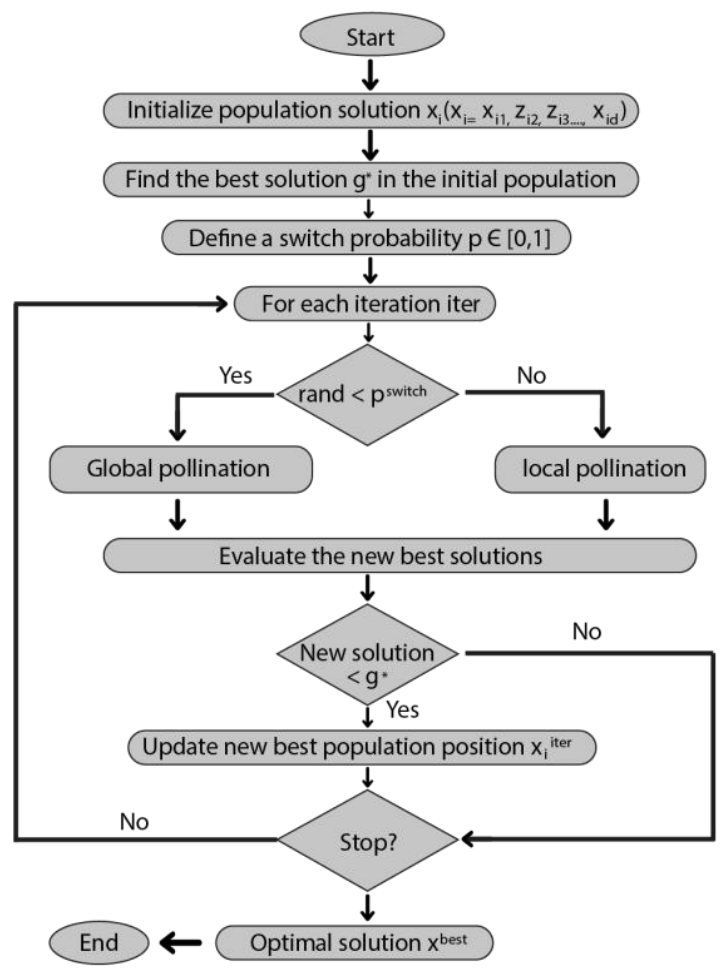

Fig 1: the flowchart of flower pollination algorithm

\section{Thresholding}

In the segmentation science, the thresholding is the simplest method. The simplest thresholding method is replacing with a black pixel if the image intensity $\mathrm{I}_{\mathrm{i} . \mathrm{j}}$ is less than constant $\mathrm{T}$. The major problem of it is that the threesholding consider only the intensity, not any relationships between the pixels. The threesholding is divided into three main types "Bi-level thresholding (Global thresholding and Variable thresholding) and Multilevel Thresholding

\subsection{Bi-level thresholding}

- Global thresholding is a simple algorithm that estimate $\mathrm{T}$ "value of threesholding "then segmentation using 
$\mathrm{T}$ if the pixel is brighter than $\mathrm{T}$ it will go to $\mathrm{G} 1$ else it will go to $\mathrm{G} 2$

$$
g(x, y)= \begin{cases}1 & f(x, y)>\mathrm{T} \\ 0 & f(x, y) \leq \mathrm{T}\end{cases}
$$

The algorithm calculates the average intensities $\mathrm{m} 1$ and $\mathrm{m} 2$ of $\mathrm{G} 1$ and $\mathrm{G} 2$ and the new threshold value $\mathrm{T}_{\text {new }}$

$T_{\text {new }}=\frac{m_{1}+m_{2}}{2}$

If $\left|T-T_{n e w}\right|>\Delta T$ the algorithm back to segment the image again with the new threshold and stop if $\left|T-T_{n e w}\right| \leq \Delta T$

Variable thresholding the variable threshold is like the global but the value of $\mathrm{T}$ can change over the image if the $\mathrm{T}$ depends on the neighborhood of $(\mathrm{x}, \mathrm{y})$ it called Local or regional thresholding and if $\mathrm{T}$ is function of $(x, y)$ it's adaptive threshold [1]

\subsection{Multilevel Thresholding}

The multiple threshold is use more than one threshold for the image segmentation the multilevel use to divided the image into background and objects it use when the image is complex and the objects are not clearly, colored images or the images with complex backgrounds [2]

$$
g(x, y)= \begin{cases}a & f(x, y)>\mathrm{T}_{2} \\ b & T_{1}<f(x, y) \leq \mathrm{T}_{2} \\ c & f(x, y) \leq T_{1}\end{cases}
$$

\subsubsection{Kapur entropy method}

An algorithm uses global and objective property of histogram, its general nature algorithm, the main concept of Kapur entropy in image segmentation is that the image is Consists of foreground and a background parts so the distribution of the intensity values can be derived the both entropy of the foreground and background are calculated than get the summation of it and maximized it the same thing can expanded to applied on the multilevel image thresholding that can be mathematically formula [3]

$$
\begin{aligned}
& H_{0}=-\sum_{t=0}^{t-1}\left(\frac{p_{i}}{\omega_{0}}\right) \log _{2}\left(\frac{p_{i}}{\omega_{0}}\right) ; \\
& H_{1}=-\sum_{t=t_{1}}^{t_{2}-2}\left(\frac{p_{i}}{\omega_{1}}\right) \log _{2}\left(\frac{p_{i}}{\omega_{1}}\right) \\
& H_{j}=-\sum_{t=t_{j}}^{t_{i+1}-1}\left(\frac{p_{i}}{\omega_{j}}\right) \log _{2}\left(\frac{p_{i}}{\omega_{j}}\right) ; \\
& H_{m}=-\sum_{t=t_{m}}^{N-1}\left(\frac{p_{i}}{\omega_{m}}\right) \log _{2}\left(\frac{p_{i}}{\omega_{m}}\right)
\end{aligned}
$$

Where

$$
\begin{array}{ll}
\omega_{0}=\sum_{t=0}^{t-1} p_{i} ; & \omega_{1}=\sum_{t=t_{1}}^{t_{2}-1} p_{i} ; \quad \omega_{j}=\sum_{t=t_{j}}^{t_{j+1}-1} p_{i} \\
\omega m=\sum_{t=t_{m}}^{N-1} p_{i} & \text { (4) }
\end{array}
$$

\section{Nature inspired Algorithms}

The nature inspired Algorithms has evolved very fast in the last years however, it is beginning work long time ago. The algorithms is depends on the social behavior of some animals or birds in the environment the study of the living phenomena and use it in the computing improve the utilization of computer the nature inspired Algorithms now days use to solve optimization problems especially the argue and complicated

one

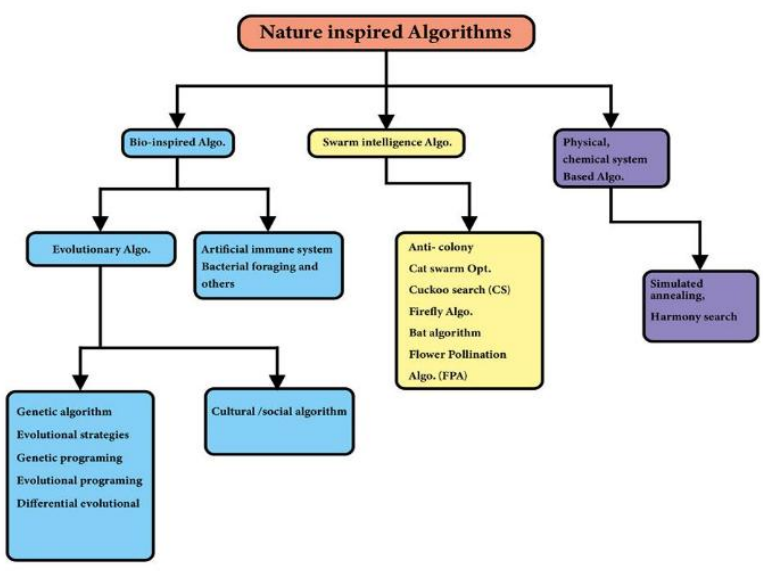

Fig 2: classification of Nature inspired Algorithms

\subsection{Flower pollination Algorithm}

The (FPA) was devolved by Xin-She Yang in 2012 inspired by the Flowering plants flow pollination process .there is four rules used in the flower pollination this rules are [4-6]

1.Global pollination involves biotic and cross-pollination where pollinators carry the pollen based on Lévy flights.

2.Local pollination involves abiotic and self-pollination.

3.Flower constancy can be considered as a reproduction probability that is proportional to the similarity between any two flowers.

4.Switch probability $P \in[0,1]$ can be controlled between local pollination and global pollination due to some external factors, such as wind. Local pollination has a significant fraction pin overall pollination activities.

The algorithm at first use random solution than it is use one of two ways to get the solution the first one is the global pollination and calculated as

$$
x_{i}^{t+1}=x_{i}^{t}+L\left(x_{i}^{t}-g^{*}\right)
$$


Where $x_{i}^{t}$ is the solution in the previous step. And $\mathrm{L}$ is drawn from a Lévy distribution "strength of the pollination".

The global optimization uses when the $\mathrm{g}^{*}$ "pollen of the flowers" is best solution

The other way is local pollination the solution is calculated by

$$
x_{i}^{t+1}=x_{i}^{t}+\varepsilon\left(x_{j}^{t}-x_{k}^{t}\right)
$$

Where $\varepsilon$ is random solution between 0 and 1 and $x_{j}^{t}, x_{k}^{t}$ are solution of separated plants

\section{Table 1: the pseudo-code of FPA}

Objective minimize or maximize $\mathrm{f}(\mathrm{x}), \mathrm{x}=(\mathrm{x} 1, \mathrm{x} 2, \ldots \mathrm{xd})$

Initialize a population of $\mathrm{n}$ flowers or pollen gametes with random numbers

Find the best solution $\left(\mathrm{g}^{*}\right)$ of the initial population

Define a switch probability $(\mathrm{p})$

For $\mathrm{t}=1: \mathrm{n}$ ( $\mathrm{n}$ is the number of iterations)

While $(\mathrm{I}<\mathrm{d})(\mathrm{d}$ is the number of flowers or pollen in the population)

If rand $<\mathrm{p}$

Global pollination Eq. (5)

Else

Local pollination Eq. (6)

\section{end if}

Evaluate new solutions

Update the better solutions in the population

\section{end While}

Find the current best solution $\left(\mathrm{g}^{*}\right)$

end For

\section{2 cuckoo search algorithm}

The cuckoo search algorithm (CS) was developed by Xin-She Yang in 2009 inspired by the behavior of some sort of cuckoo birds that laying its leg on the other nests. Three rules are control the cuckoo search this rules are $[4,7,8]$

1.Each cuckoo lays one egg at a time, and dumps its egg in a randomly chosen nest.
2. The best nests with high quality of eggs will carry over to the next generation.

3.The number of available hosts nests is fixed, and the egg laid by a cuckoo is discovered by the host bird with a probability $P_{a} \in[0,1]$ (switch probability) Discovering operate on some set of worst nests, and discovered solutions dumped from farther calculations.

And for the CS the random walk is better than the Lévy flights.

Like the most of the Nature inspired algorithms the algorithm starts with generated random solution calculated as

$x_{i, j}=x_{j}^{\min }+\operatorname{rand}(0,1)\left(x_{j}^{\max }-x_{j}^{\min }\right)$

From the rule number three the host may be discovering that the egg is a cuckoo egg (rejected solution) so the algorithm mill replacing it with new solution the new solution will be calculated using Lévy flights by the equation

$x_{i}(t+1)=\mathcal{X}_{i}(t)+\alpha \oplus \operatorname{Lévy}(\lambda)$

In this equation $\alpha$ is the step size. And the step size in the Lévy distribution calculated by

$$
\operatorname{Lévy}(\lambda)=t^{-\lambda} ; 1<\lambda \leq 3
$$

The variance of Lévy flights is calculated by

$$
\sigma^{2}(t) \sim t^{2-\beta} ; 1 \leq \beta \leq 2
$$

This iteration repeated until reaching the global optimization

\section{Table 1: the pseudo-code of cuckoo search}

Table 2: the pseudo-code of cuckoo search

$$
\begin{aligned}
& \text { Objective function } f(x), x=\left(x_{1}, x_{2}, \ldots \ldots \ldots, x_{n)}\right. \\
& \text { Generate an initial population of n host nests } \\
& \text { While (stop gauge) } \\
& \text { Get a cuckoo randomly, create new solutions using Lévy } \\
& \text { flights } \\
& \text { Evaluate solutions quality/fitness } F_{i} \\
& \text { Choose random solution (nest) } F_{j}
\end{aligned}
$$


If $\left(F_{i}>F_{j}\right)$

Replace J by the new solution

\section{End IF}

Append the worst nest with probability $P_{a}$

\section{Build new nest}

Evaluate fitness and rank the solution a find the current best and pass it to the next generation

\section{End while}

\subsection{CS McCulloch algorithm}

An algorithm proposed in 2016 it's an modification to the CS the algorithm using the following equations and pseudo code [9]

The Population Initialization like the Artificial bee colony algorithm $\mathrm{ABC}$ with the equation

$x_{i, j}=x_{j}^{\min }+\operatorname{rand}(0,1)\left(x_{j}^{\max }-x_{j}^{\min }\right)$

The step size in levy flight is calculating using the following equation

$$
s_{z}=c\left[\frac{\cos ((1-\alpha) \phi)}{\omega}\right]^{\frac{1}{\alpha}-1}\left[\frac{\sin (\alpha \phi)}{\cos (\phi)}\right]^{\frac{1}{\alpha}}-\delta(12)
$$

And the replacement of the worst nest is use equation

$$
x_{i}(t+1)=x_{i}(t)+\alpha \oplus \operatorname{lev} y(\lambda)
$$

Table 3: the pseudo-code $3.3 \mathrm{CS}_{\text {McCulloch }}$

Population Initialization via equation (11)
Objective function $f(x), x=\left(x_{1}, x_{2}, \ldots \ldots \ldots, x_{n)}\right.$
If (ITER $<$ MAXITER) then
Generate new solution space by retaining the current best;
Fitness value computation; Memorize best nest
If k $<P_{a}$

Step size $(\alpha)$ generation using equation (12)

Replace worst nest using equation (13)
Fitness value computation; Memorize best nest;
Update the Counter;
Find best fitness value so far;
Retain those nests;
end
end
Find the optimum solutions;

\section{The experiment}

\section{1 image choosing}

in these research we choose two images from satellite one is colored image to city of Shanghai in china and the other id gray level image for Kabul airport in Afghanistan the images from Pléiades Satellite Imagery
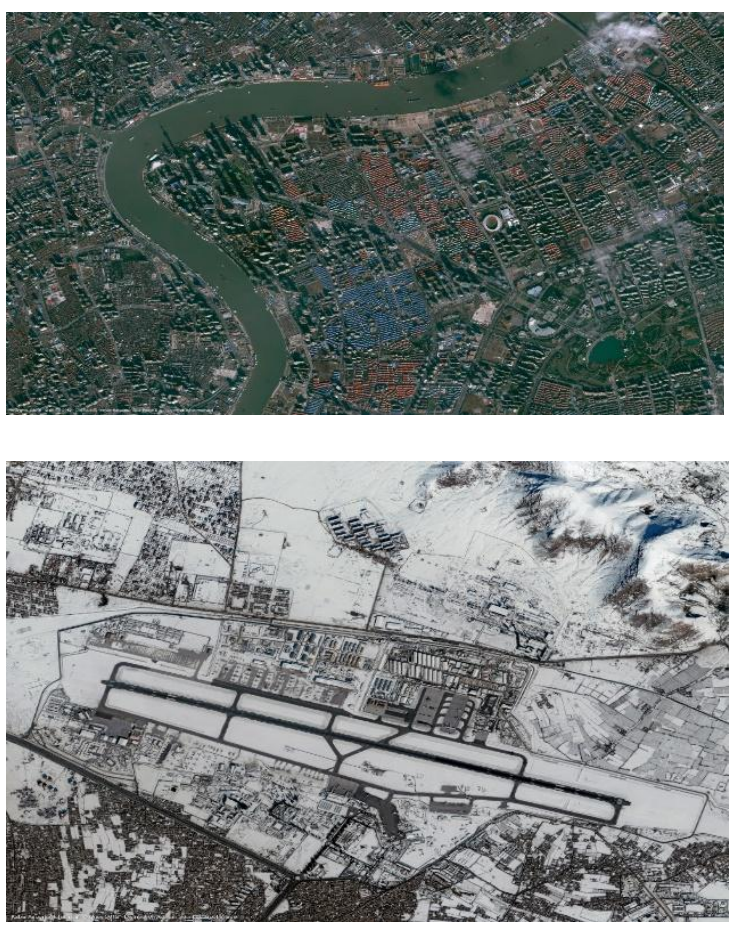

Fig 3: two different satellite images used in the study: Ple'iades Satellite Images: a) sat 1: Shanghai, China; b) sat 2: Kabul Airport 

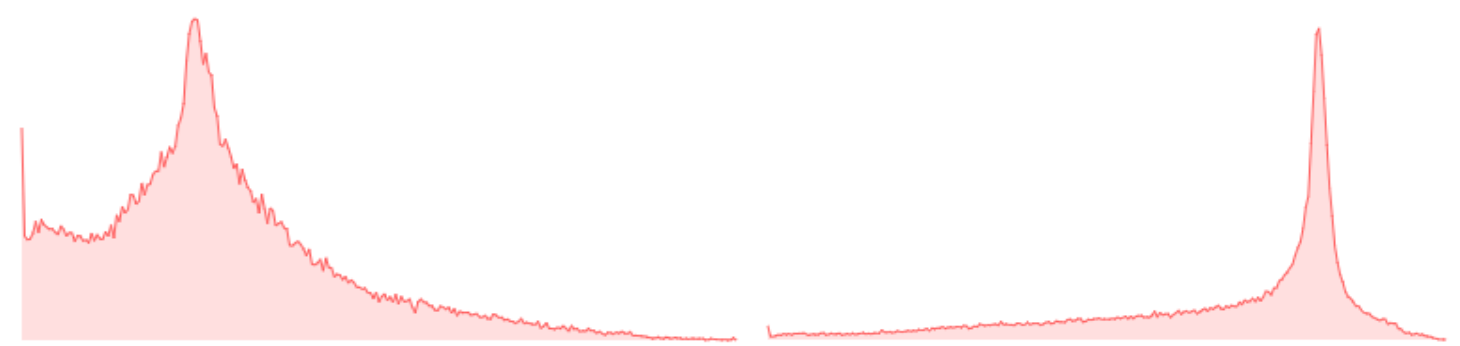

Fig 4: the histogram of the images "calculated using pinetools.com"

\section{2 the results}

$N=5$

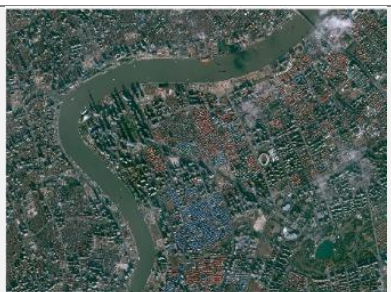

$C S_{\text {Mc Culloch }}$

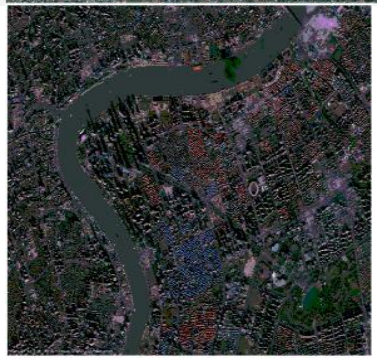

$N=7$
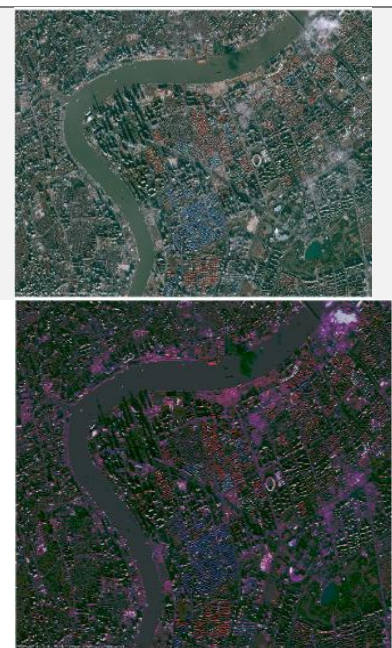

$N=9$
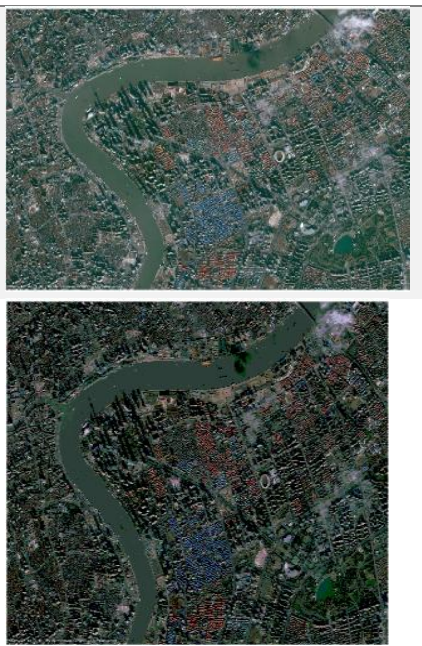

$N=11$
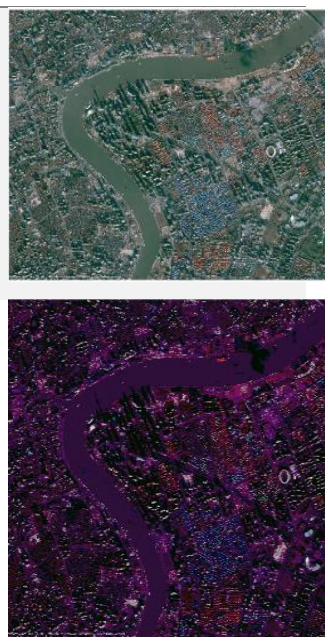

Fig 5: the result of segmentation using Kapur entropy method for shanghai, China image using FPA and CS

$\mathrm{N}=5$

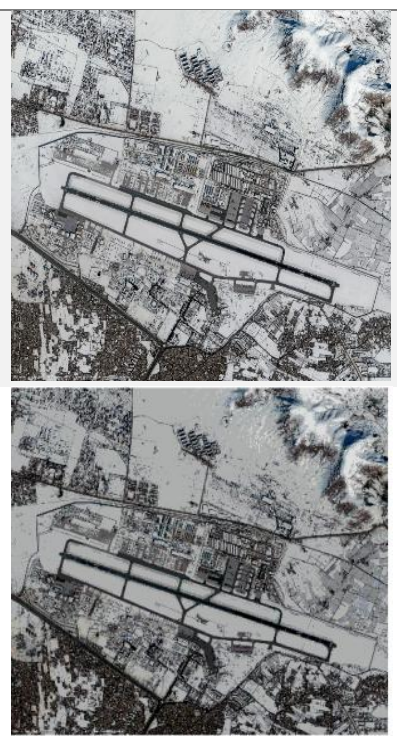

$$
\mathrm{N}=7
$$
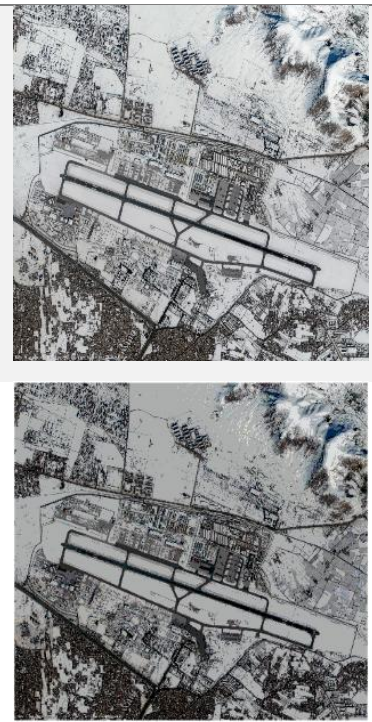

$\mathrm{N}=9$
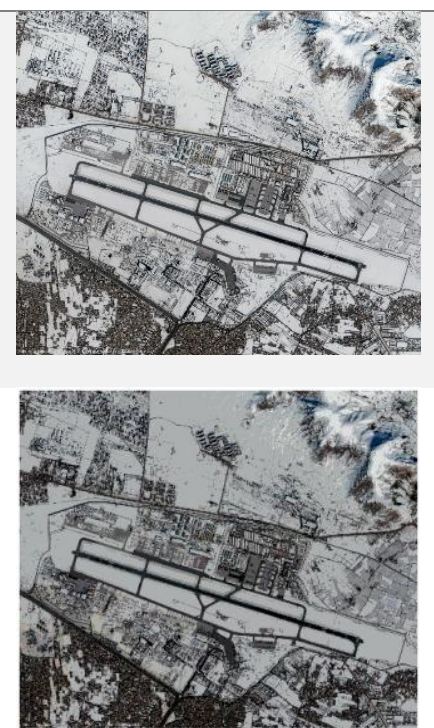

$\mathrm{N}=11$
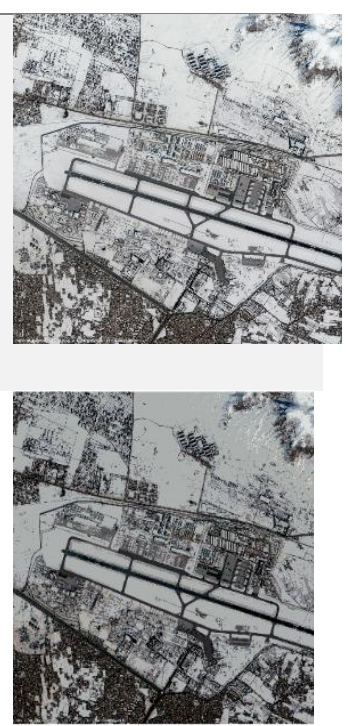

Fig 6: the result of segmentation using Kapur entropy method for Kabul Airport image using FPA and CS 


\section{3 the PSNR comparison}

Table 4: PSNR value comparison "calculated by PSNR.exe from gipsa-lab.grenoble"

\begin{tabular}{|c|c|c|c|}
\hline Image & $\mathrm{n}$ & FPA & $\mathrm{CS}_{\text {McCulloch }}$ \\
\hline \multirow{4}{*}{$\begin{array}{l}\text { shanghai, } \\
\text { China }\end{array}$} & 5 & 13.54 & 13.36 \\
\hline & 7 & 13.40 & 13.80 \\
\hline & 9 & 13.25 & 13.35 \\
\hline & 11 & 13.71 & 13.51 \\
\hline \multirow{4}{*}{ Kabul Airport } & 5 & 29.35 & 29.30 \\
\hline & 7 & 26.58 & 26.64 \\
\hline & 9 & 25.43 & 24.85 \\
\hline & 11 & 22.86 & 22.51 \\
\hline
\end{tabular}

\section{4 the running time}

Table 5: running time comparison "code run by mat lab 2017 on windows 10 64-bit ,8G RAM and core i7-3517U CPU"

\begin{tabular}{|c|c|c|c|}
\hline Image & $\mathrm{n}$ & FPA & $\mathrm{CS}_{\text {McCulloch }}$ \\
\hline \multirow{4}{*}{$\begin{array}{l}\text { shanghai, } \\
\text { China }\end{array}$} & 5 & $1.371 \mathrm{~s}$ & $1.407 \mathrm{~s}$ \\
\hline & 7 & $1.533 \mathrm{~s}$ & $1.543 \mathrm{~s}$ \\
\hline & 9 & $1.793 \mathrm{~s}$ & $1.784 \mathrm{~s}$ \\
\hline & 11 & $1.834 \mathrm{~s}$ & $1.865 \mathrm{~s}$ \\
\hline \multirow{4}{*}{ Kabul Airport } & 5 & $1.351 \mathrm{~s}$ & $1.295 \mathrm{~s}$ \\
\hline & 7 & $1.471 \mathrm{~s}$ & $1.559 \mathrm{~s}$ \\
\hline & 9 & $1.551 \mathrm{~s}$ & $1.575 \mathrm{~s}$ \\
\hline & 11 & $1.795 \mathrm{~s}$ & $1.839 \mathrm{~s}$ \\
\hline
\end{tabular}

\section{CONCLUSION}

However, CS McCulloch algorithm have advantage on PSO, DPSO, ABC and CS that the algorithm have execute time complexity lower then all of them when increase the threshold put the FPA have little time then the CS McCulloch and the PSNR of the image from the FPA is better than the output of CS McCulloch the FPA has another advantage because its total running time is often little than the total running time of $\mathrm{CS}_{\mathrm{McCulloch}}$

\section{ACKNOWLEDGMENTS}

The authors would like to thank the anonymous reviewers for their valuable suggestions and comments which helped to improve the quality of the research paper. Also we would like to thank prof Yang, X.-S. for his efforts and researches in Nature-Inspired Metaheuristic Algorithms

\section{REFERENCES}

[1] Cheng, H.-D., et al., Color image segmentation: advances and prospects. Pattern recognition, 2001. 34(12): p. 2259-2281

[2] Romshoo, S.A. and I. Rashid, Assessing the impacts of changing land cover and climate on Hokersar wetland in Indian Himalayas. Arabian Journal of Geosciences, 2014. 7(1): p. 143-160

[3] Yang, X.-S., Nature-inspired metaheuristic algorithms. 2010: Luniver press.

[4] Yang, X.-S. Flower pollination algorithm for global optimization. in International conference on unconventional computing and natural computation. 2012. Springer

[5] Davies, E., Stable bi-level and multi-level thresholding of images using a new global transformation. IET Computer vision, 2008. 2(2): p. 60-74.

[6] Arora, S., et al., Multilevel thresholding for image segmentation through a fast statistical recursive algorithm. Pattern Recognition Letters, 2008. 29(2): p. 119-125.

[7] Kapur, J.N., P.K. Sahoo, and A.K. Wong, A new method for gray-level picture thresholding using the entropy of the histogram. Computer vision, graphics, and image processing, 1985. 29(3): p. 273-285.

[8] Nigdeli, S.M., G. Bekdaş, and X.-S. Yang, Application of the flower pollination algorithm in structural engineering, in Metaheuristics and optimization in civil engineering. 2016, Springer. p. 25-42.

[9] Yang, X.-S. and S. Deb. Cuckoo search via Lévy flights. in Nature \& Biologically Inspired Computing, 2009. NaBIC 2009. World Congress on. 2009. IEEE.

[10] Lim, W.C.E., G. Kanagaraj, and S. Ponnambalam, PCB drill path optimization by combinatorial cuckoo search algorithm. The Scientific World Journal, 2014. 2014.

[11] Suresh, S. and S. Lal, An efficient cuckoo search algorithm based multilevel thresholding for segmentation of satellite images using different objective functions. Expert Systems with Applications, 2016. 58: p. 184-209. 